\title{
Research on the Band Gap Characteristics of Two-Dimensional Phononic Crystals Microcavity with Local Resonant Structure
}

\author{
Mao Liu, ${ }^{1,2}$ Pei Li, ${ }^{1}$ Yongteng Zhong, ${ }^{1}$ and Jiawei Xiang' \\ ${ }^{1}$ College of Mechanical \& Electrical Engineering, Wenzhou University, Wenzhou 325035, China \\ ${ }^{2}$ School of Mechanical and Electrical Engineering, Guilin University of Electronic Technology, Guilin 541004, China \\ Correspondence should be addressed to Jiawei Xiang; jwxiang@wzu.edu.cn
}

Received 22 October 2014; Accepted 30 November 2014

Academic Editor: Yumin He

Copyright (c) 2015 Mao Liu et al. This is an open access article distributed under the Creative Commons Attribution License, which permits unrestricted use, distribution, and reproduction in any medium, provided the original work is properly cited.

\begin{abstract}
A new two-dimensional locally resonant phononic crystal with microcavity structure is proposed. The acoustic wave band gap characteristics of this new structure are studied using finite element method. At the same time, the corresponding displacement eigenmodes of the band edges of the lowest band gap and the transmission spectrum are calculated. The results proved that phononic crystals with microcavity structure exhibited complete band gaps in low-frequency range. The eigenfrequency of the lower edge of the first gap is lower than no microcavity structure. However, for no microcavity structure type of quadrilateral phononic crystal plate, the second band gap disappeared and the frequency range of the first band gap is relatively narrow. The main reason for appearing low-frequency band gaps is that the proposed phononic crystal introduced the local resonant microcavity structure. This study provides a good support for engineering application such as low-frequency vibration attenuation and noise control.
\end{abstract}

\section{Introduction}

In recent years, a growing interest has been focused on the study of the propagation of elastic waves in the periodic phononic crystals (PCs) [1-4]. PCs composed of two or more materials with different mechanical properties will generate band gaps (BGs) within which the propagation of elastic or acoustic waves is prohibited. The great attention on BGs is mainly due to their rich physical properties and potential applications in the design of acoustic devices such as noise reduction, waveguides, and acoustic filters $[5,6]$.

In order to promote the application of phononic crystals in the fields of vibration control and noise isolation, obtaining tunable BGs with large bandwidth in the low-frequency domain is significant. In the 2DPCs, a lot of studies on the research of large band gap have been carried out to obtain PC structures with excellent BGs. For example, Lai et al. have studied the PC structure composed of the square lattice of steel cylinders in air background and found that the BGs could be tunable with various microstructures [7]. Wang et al. have introduced the narrow slit structures into the inclusions of the two-dimensional PC structure composed of steel tubes in air and obtained large BGs in audible frequency range. Wang et al. discussed the absolute band gap (ABG) structures in PCs with cross-like holes [8]. Liu et al. designed a tree-component PC structure that exhibits BGs two orders of magnitude smaller than the relevant wavelength which proposed the localized resonance (LR) mechanism [9]. As the LRPCs allowed the acquisition of low-frequency band gaps without considerably increasing the size of the structures, they encouraged more extensive application prospects and attracted more and more attention.

Recently, some novel periodic structures with large band gaps are reported. Trabelsi et al. investigated the band properties of a phononic crystal composed of alternating fluid and fluid-saturated porous layers [10]. They observed lowfrequency band gaps of underwater structure. Manktelow et al. studied the nonlinear periodic materials [11] and obtained large band gap shifts in one and two dimensions via optimal topologies. Salehi et al. researched the propagation of acoustic waves in the phononic crystal of 3D with rhombohedral lattice [12]. Sainidou and Stefanou analyse guided and quasiguided elastic waves in a thin glass slab coated on one side with a periodic monolayer of polymer spheres, immersed 


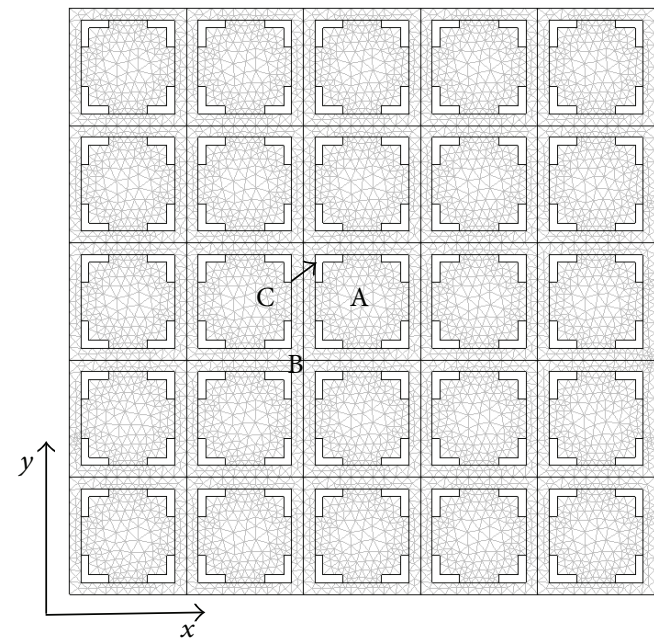

(a)

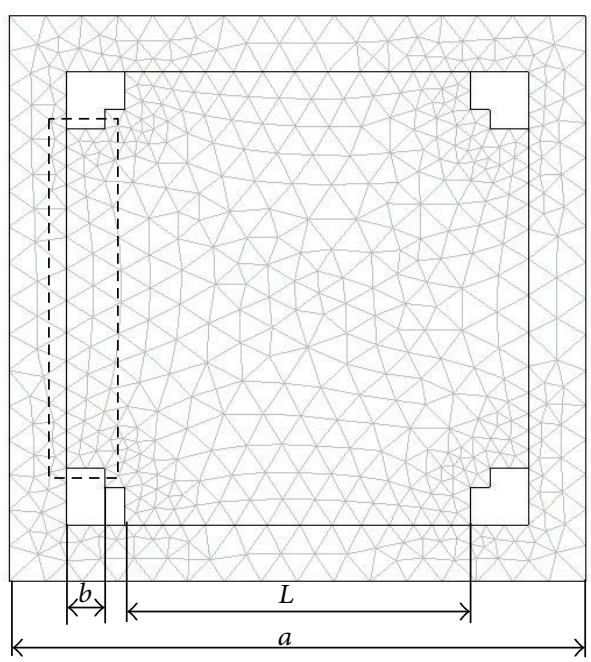

(b)

FIGURE 1: The cross-section structure of the phononic crystal (a) and the unit cell structure of the phononic crystal (b).

in water [13]. Oudich et al. researched the band gaps in the PC structure constructed by periodically depositing single-layer or two-layer stubs on the surface of a thin plate and proposed a "spring-mass" model to explain the opening of the LR band gaps [14]. Yu et al. studied 2DPCs with neck structures and obtained the large band gaps in the low frequency [15]. Wang et al. also explained the ternary locally resonant PCs with a comb-like coating using "spring-mass" model [8].

In this paper, new band gaps structure composed of square scatter with microcavity structure embedded in a homogeneous matrix is considered. We adopted the finite element method to investigate the variable of the band gaps in low frequency. Different numbers of connection bridge plates and connection forms will be studied to confirm the locally resonant mechanism. Furthermore, microcavity structures with different geometric dimension are studied in this work. Finally, some conclusions are given.

\section{Model and Method of the Calculation}

PC structure considered here is a square lattice unit cell which contains a square inclusion and junction plates embedded in a homogeneous matrix. Figures 1(a) and 1(b) illustrate the schematic view of the cross-sections of the PC structure and one of the unit cells. Figures $1 \mathrm{~A}, 1 \mathrm{~B}$, and 1C denote the scatter, the matrix, and the microcavity, respectively. The extended direction is selected along the $x$ - and $y$-axes. As shown in Figure 1(b), the microcavity structure falls in between scatter and matrix. Square scatter is connected to the matrix via 4 connection bridge plates (in dashed box shown in Figure 1(b)) which are the same materials with scatter. The length and width of the connection bridge plates are $L$ and $b$, respectively. The lattice spacing of the square constant is $a$.

In the present study, to research the band gap characteristics of the proposed 2DPC structure, a lot of computes on the dispersion relations and transmission spectra are performed with the FEM [16]. For the calculations of the band structures of the proposed PCs, the considered structure refers to an infinite system. The governing field equations for elastic wave propagation in solids are given by

$$
\sum_{j=1}^{3} \frac{\partial}{\partial x_{j}}\left(\sum_{l=1}^{3} \sum_{k=1}^{3} c_{i j k l} \frac{\partial u_{k}}{\partial x_{l}}\right)=\rho \frac{\partial^{2} u_{i}}{\partial t^{2}} \quad(i=1,2,3),
$$

where $\rho$ is the mass density, $c_{i j k l}$ are the elastic constants, $u_{i}$ is the displacement, $t$ is the time, and $x_{j}(j=1,2,3)$ denotes the coordinate variables $x, y$, and $z$, respectively. Because the infinite system is periodic along the $x$ - and $y$-directions simultaneously, only the unit cell shown in Figure 1(b) is taken into account, based on the Bloch theorem. With the FEM, the Bloch theorem is adopted through the implementation of the Bloch periodic boundary conditions on the mentioned boundaries of the unit cell. The periodic boundary conditions on the two group boundaries of the unit cell yield

$$
\mathbf{U}(\mathbf{r}+\mathbf{a})=e^{i(k \cdot \mathbf{a})} \mathbf{U}(\mathbf{r}),
$$

where $\mathbf{r}$ is located at the boundary nodes, $\mathbf{k}$ is wave vectors, $\mathbf{U}$ is the displacement at the nodes, and $\mathbf{a}$ is the vector that generates the point lattice associated with the phononic crystals. As the angular frequency $\omega$ is a periodical function of the wave vector, the problem can be reduced to the first Brillouin zone. The dispersion curves are eventually built by varying the wave vector $\mathbf{k}=\left(k_{x}, k_{y}\right)$ on the first Brillouin zone for a given propagation direction. The full band structure as well as the eigenmodes of the structure is then deduced using symmetries.

For the analysis of the transmission spectra, a finite system must be defined. We consider here the structure being finite in the $x$-direction that contains $N$ units. In the $y$ direction are $M$ units; the periodic boundary conditions are 


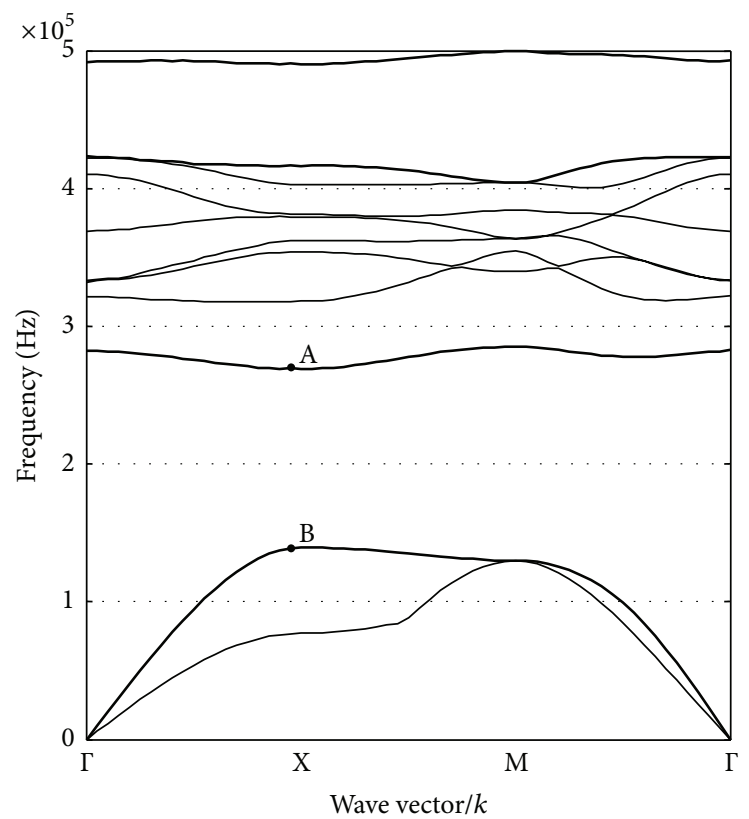

(a)

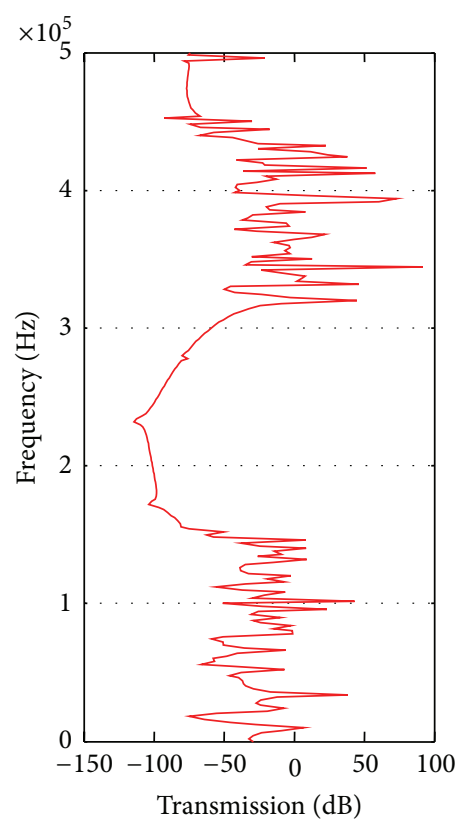

(b)

FIGURE 2: The dispersion curves of the phononic crystal with microcavity structure (a) and the transmission spectra of the phononic crystal with microcavity (b).

still utilized to represent the infinite units. In this case, a finite array structure composed of $N \times M$ units is modeled for the calculations. The plane waves with single frequency, supplied by the acceleration excitation source, are incident from the left side of the finite array and propagate along the $x$-direction, and the corresponding transmitted acceleration value is recorded on the right side. The transmission is defined as

$$
T L=20 \log _{10}\left(\frac{v_{o}}{v_{i}}\right),
$$

where $v_{o}$ and $v_{i}$ are the value of the transmitted and the incident displacement, respectively. Then, by varying the excitation value of the incident displacement, the transmission spectra can be obtained.

\section{Numerical Examples and Discussion}

In this section, the proposed 2DPCs with microcavity structures will be studied using the finite element software COMSOL. The detailed process can be found in [17]. The wave vectors are restricted within the $x-y$ plane and point to the boundary ГХМГ of the irreducible first $\mathrm{BZ}$ of the square lattice structure. Based on the Bloch theorem, this is sufficient to determine the complete frequency band structures. The material parameter of two kinds of materials is shown in Table 1. The following structure parameters are used: $L=$ $0.001 \mathrm{~m}$ and $b=0.0002 \mathrm{~m}$, and the square lattice constant is $a=0.003 \mathrm{~m}$.

We observe that bands are contained in the frequency range of $0-500 \mathrm{kHz}$, in Figure 2(a), where three complete
TABLE 1: Materials properties.

\begin{tabular}{lccc}
\hline Materials & $\begin{array}{c}\text { Density }(\rho) \\
\left(\mathrm{Kg} / \mathrm{m}^{3}\right)\end{array}$ & $\begin{array}{c}\text { Young's modulus }(E) \\
\mathrm{Pa}\end{array}$ & Poisson's ratio $(\nu)$ \\
\hline $\mathrm{A}$ & 4.08 & 11600 & 0.369 \\
$\mathrm{~B}$ & 0.435 & 1180 & 0.368 \\
\hline
\end{tabular}

band gaps are involved. The lowest one ranges from 14.93 to $26.92 \mathrm{kHz}$ (between the second and third band), while the second and third band gaps are extended from 28.26 to $31.76 \mathrm{kHz}$ (between the third and fourth bands) and 42.29 to $49.02 \mathrm{kHz}$ (between the tenth and eleventh bands), respectively. In order to verify the calculations of the band gaps, the transmission spectra through the PC structure composed of a finite array of $5 \times 5$ units are computed. As shown in Figure 2(b), clearly existing a frequency range extending from 14.93 to $32 \mathrm{kHz}$ in which the attenuation is so large, thus it can be treated as a complete band gap. The starting and terminating frequencies of the band structure coincided well with the result of band gap compute using FEM in Figure 2(a), validating the numerical results of the band structure calculations. The appearance of the two improving frequency ranges in the band structure, which correspond precisely to the third and fourth bands in the band gap, is mainly owed to the existence of the localized modes.

As a comparison, we also compute the gap characteristics of the traditional PC composed of scatter inclusions embedded in the epoxy matrix with full contact, namely, no microcavity structure. Obviously, it is a classic 2DPC structure based on the Bragg scattering mechanism. During 


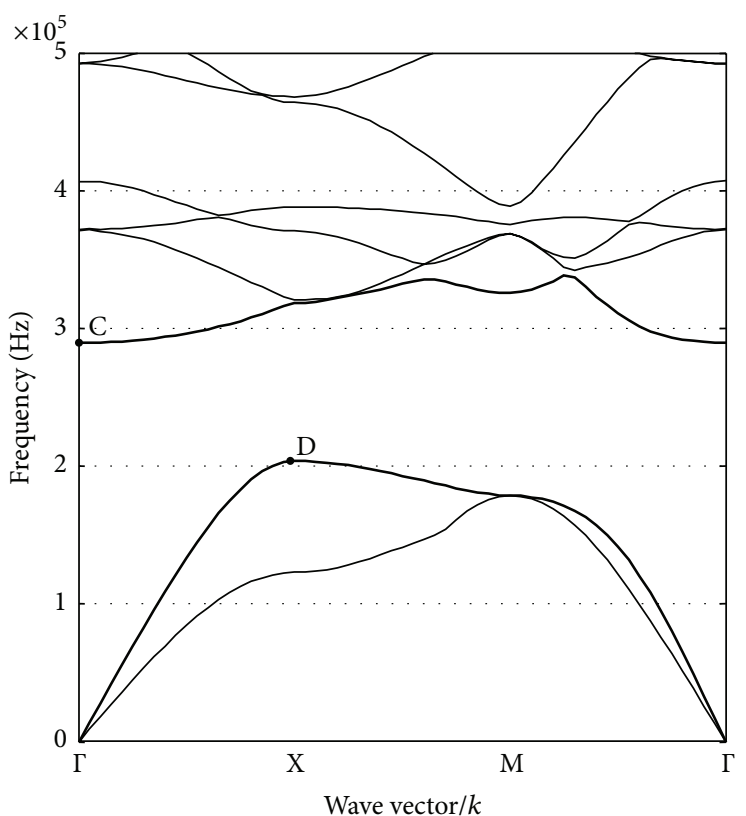

(a)

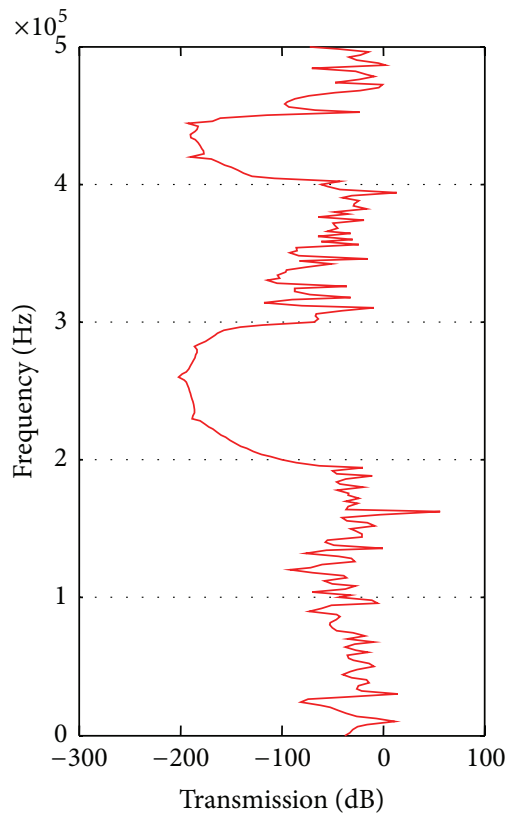

(b)

FIgURE 3: The dispersion curves of the phononic crystal with no microcavity structure (a) and the transmission spectra of the phononic crystal with no microcavity (b).

the computations, the lattice constant and filling ratio are selected to be the same as those used in Figure 2. The results are shown in Figure 3. It can be revealed that there are few bands in the frequency range of $0-500 \mathrm{kHz}$. The only band gap locates upon the second band, with starting frequency $20.34 \mathrm{kHz}$. At the same time, the transmission spectra of the finite array of $5 \times 5$ units are calculated to confirm the analysis of the band structure. The results are shown in Figure 5; the attenuation frequency range of the acceleration responses coincides well with the frequency range that parallels the band gap. Comparing the structures with microcavity, we can discover that the frequency range of the band structure in PC with microcavity structures is lower at the lower edge of band gap than that of the PCs having no microcavity structures; at the same time, the width of the gap is extremely large compared to classic PC structures and meanwhile the whole weight of the microcavity structures is lighter via some material will removed. All these excellent characteristics will promote the applications of these new microcavity PC structures in the low-frequency noise control in engineering field and the design of the low-frequency reductions.

In order to confirm the physical mechanism for the occurrence of the low-frequency band structures in the proposed PC structure, we compute the eigenmode shapes and displacement vector fields at the edges of the first complete band gap. The results are shown in Figure 4 and the behavior is discussed in the following. The color maps of Figure 4 are the magnitude of the total displacement vector field. The modes on the lower and upper edges of microcavity structure are discussed first. It can be obviously observed that the vibration of mode A, which corresponds to the lower edge of the first band structure, is mainly concentrated at the square scatter and four connection bridge plates, while the matrix nearly remains motionless. The connection bridge plates extrude the matrix with the result that localized large deformation. The vibration process can be seen as a mass-spring system. The square scatter works as a resonator and the connection bridge plates play the role of the spring. With different mode A, the vibration of mode $B$ is mainly the torsion of the matrix and meanwhile the out-of-phase vibration of the matrix. The square scatter and the connection bridge plates remain static. Because the vibration direction of the matrix is outward, the square scatter and the connection bridge plates remain localized. Then, we investigated the modes on the lower and upper edges of classic PC structure. From Figures 4(c) and 4(d), corresponding to the structure vibration of modes $\mathrm{C}$ and $\mathrm{D}$ are mainly extrudes and compresses the matrix; that is to say, there have been no localized conditions.

From the analysis above, we can conclude that the occurrence of the large band gaps in low frequency is mainly attributed to the existence of the localized resonance modes resulting from the introduction of the four connection bridge plates formed microcavity structures. Since the connection bridge plates play the role of springs linking the square scatter and the matrix, their shape features may influence the band structure remarkably. In the following, we will study the effects of the geometrical parameters on the typical large lowfrequency band gaps of the proposed PC structure.

Furthermore, we also investigated the band gaps of microcavity structures with 1,2 , and 3 connection bridge plates to the matrix, respectively. The calculated dispersion curves are shown in Figure 5. The variation of the number 


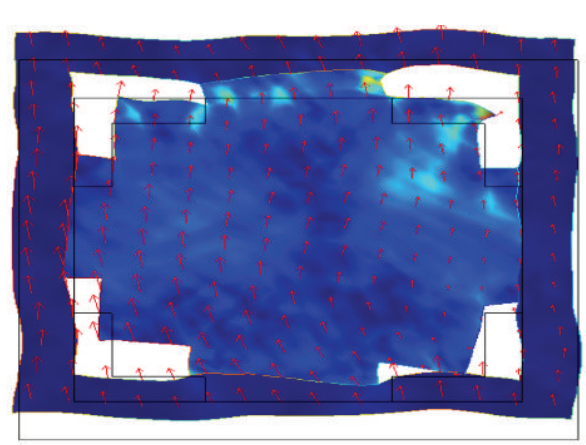

(a)

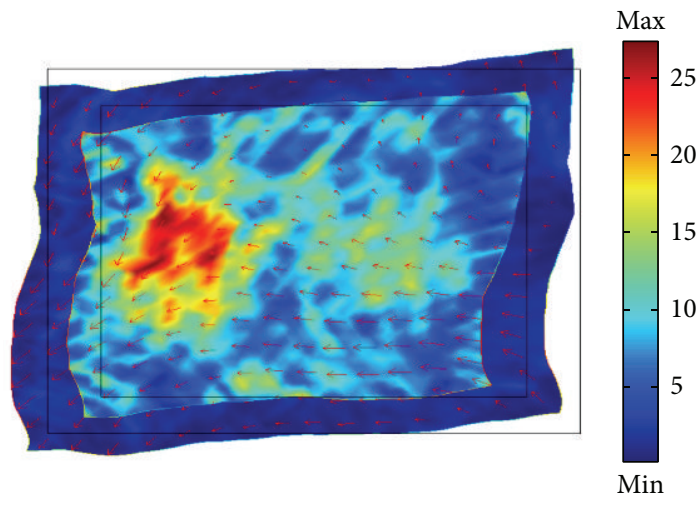

(c)
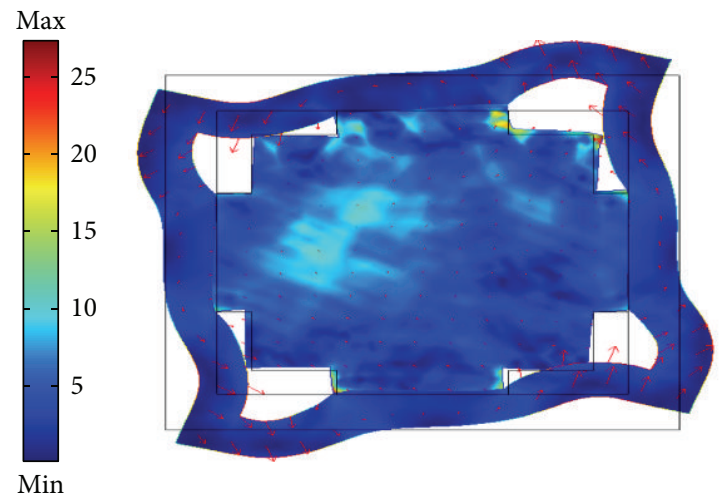

(b)

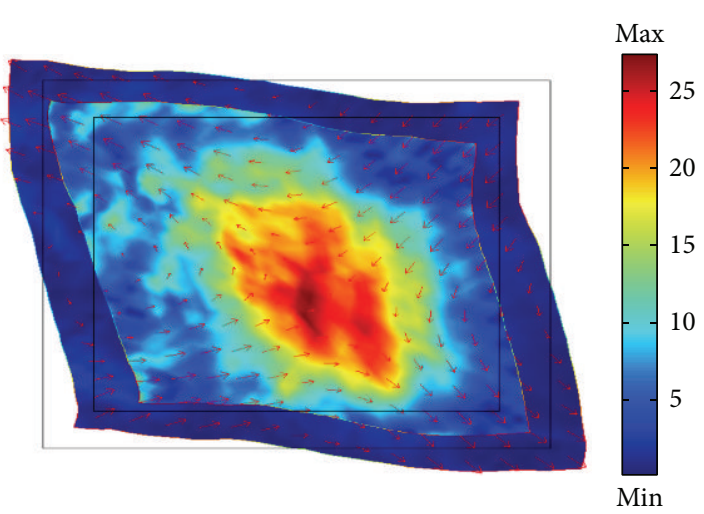

(d)

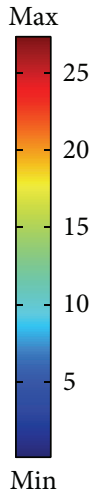

25

5

Min

Figure 4: Eigenmodes shapes and displacement vector fields of the modes marked in Figures 2 and 3 correspond to points A, B, C, and D, respectively.

of connection bridge plates, however, does not significantly influence the band structures, and they show for all four scatter shapes very similar features. We can observe clearly existing incomplete band gaps X-M direction of the irreducible first Brillouin zone in Figures 5(a) and 5(b). Because of these two connection shapes, a connection plate plays a role of the spring extruding the resonator and another connection plate works as a spring to compress the resonator. So the vibration process can be seen as two mass-spring systems with out-ofphase vibration which leads to attenuation of frequency in X$M$ direction. In addition, because the direction of vibration on connection plates is not consistent in Figures 5(c) and 5(d), band gaps of these structures do not exist. This is slightly surprising and indicated that the connection form provides a more important design factor.

3.1. Effect of the Length of Connection Plates on the LowFrequency Band Gap. The objective of the following is to investigate the effects of geometric dimension on the lowfrequency band gap with microcavity structure. First, we investigate the influence of the length of the connection bridge plates on the band gap. Keeping the lattice constant $a=3 \mathrm{~mm}$, the thickness of the matrix is $0.3 \mathrm{~mm}$ and the width of connection bridge plate $b=1 \mathrm{~mm}$. The results are shown in Figure 6. We can observe that the lower edges of the first band gap move to a higher frequency range with the increase of the length of the connection bridge plates $L$ from 0.2 to $1.8 \mathrm{~mm}$, while the variable is minor. At the same time, the upper edges of the first band gap are larger and the increase of the frequency of the upper edge is faster than that of the lower edge. In fact, the length of the connection plates influences not only the frequency range of the band gap but also the value of the attenuation in the gap.

\subsection{Effect of the Width of Connection Plates on the Low-} Frequency Band Gap. Second, we investigate the influence of the width of connection plates on the band structure. Figure 7 displays the evolution of the band gap as a function of the width $b$ with $a=3 \mathrm{~mm}$ and $L=1.4 \mathrm{~mm}$ and the thickness of the matrix is $0.3 \mathrm{~mm}$, the upper edge (black dashed line with square points) and lower edge (black solid line with circle points). It can be observed that, with the width of the connection plates increasing from 1.4 to $1.8 \mathrm{~mm}$, the upper edge of the band gap rises rapidly from 305.4 to $318.4 \mathrm{kHz}$, while the lower edge of the band gap declines slightly. We can see that the width of the connection plates mainly affects the upper edge of the band gap and has limited effect on the lower edge. For the upper edge, with the increase of the width, the stiffness of the connection plates in the massspring system increases rapidly, quicker than that of the mass 


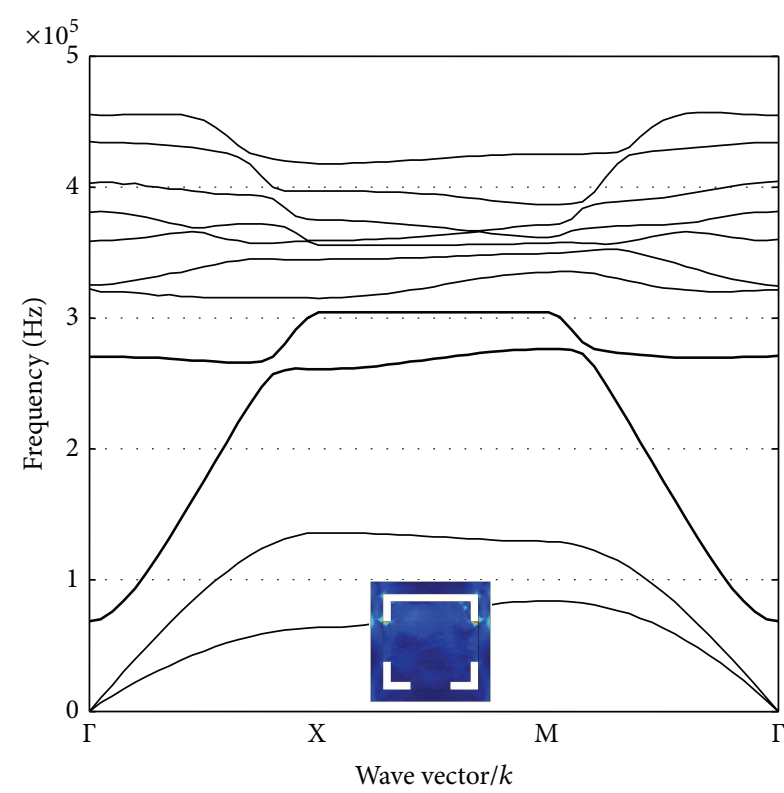

(a)

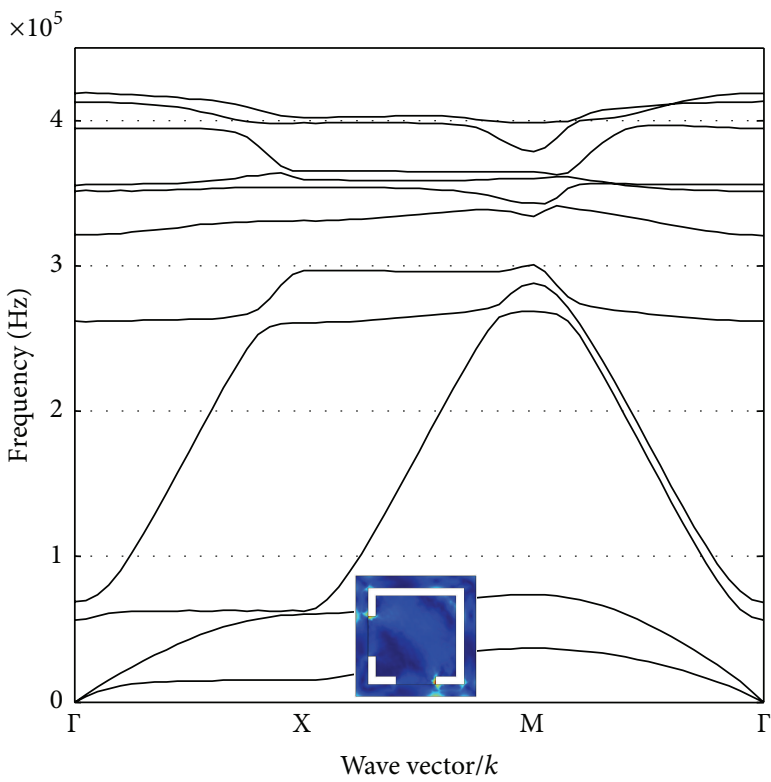

(c)

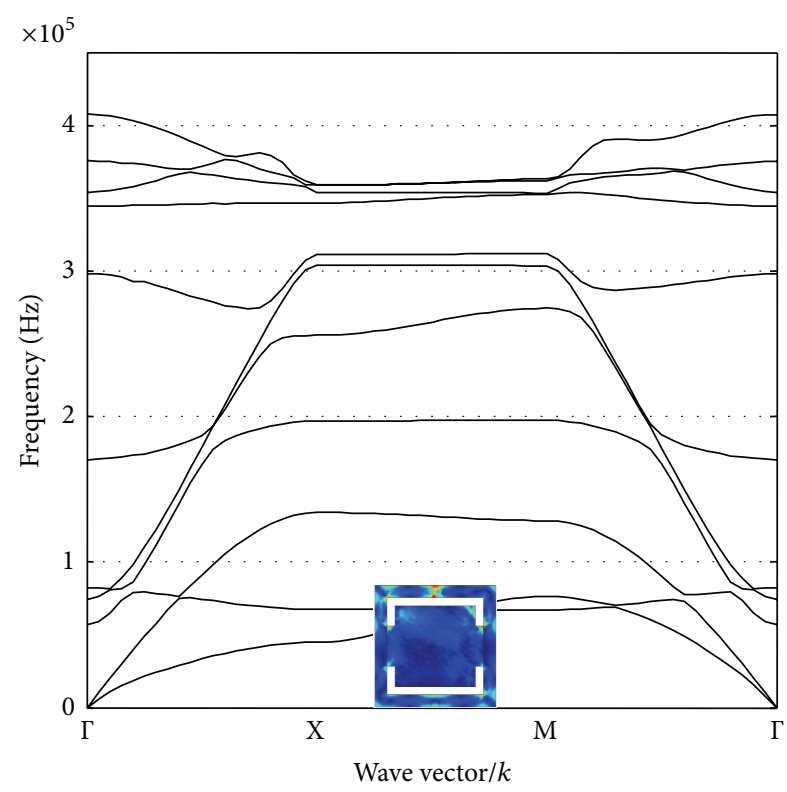

(b)

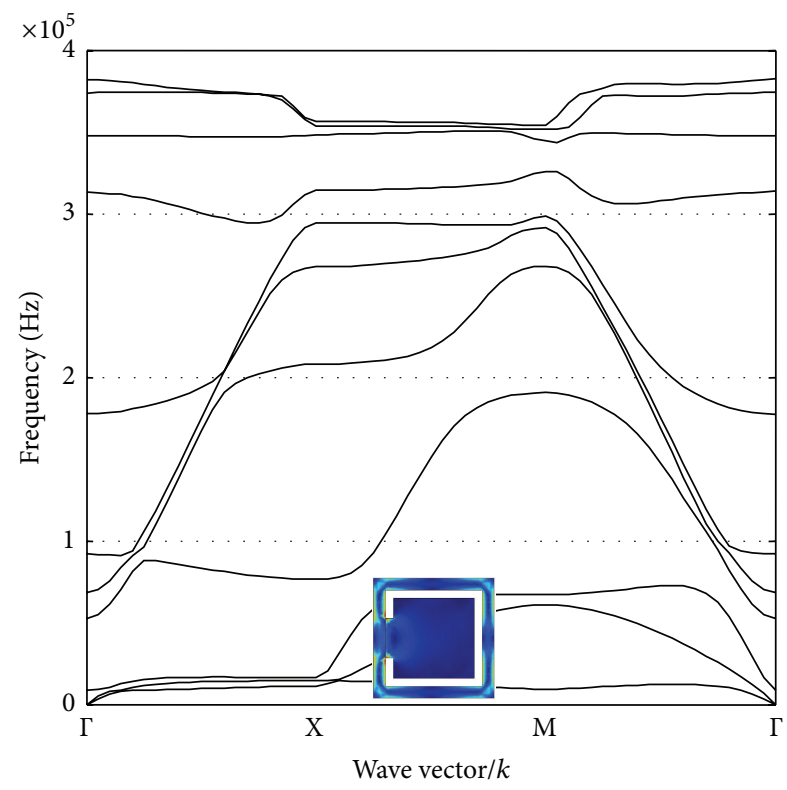

(d)

FIGURE 5: The dispersion curves of the phononic crystal with three connection plates (a). The dispersion curves of the phononic crystal with two opposite connection plates (b). The dispersion curves of the phononic crystal with two adjacent connection plates (c). The dispersion curves of the phononic crystal with only one connection plate.

of the resonator, resulting in the resonance frequency rising accordingly. For the lower edge, whose relevant mode is concentrated on the matrix, the increase of the width has little influence on relevant mode.

3.3. Effect of the Density of Connection Plates on the LowFrequency Band Gap. Finally, we also research the effect of the density of the connection plates on the band gaps. Figure 8 shows the evolution of the band gap as a function of the density with four connection plates; setting $a=3 \mathrm{~mm}$, $L=10 \mathrm{~mm}$, and $b=2 \mathrm{~mm}$, the red, black, and blue rectangular boxes denote the first, second, and third band gap, respectively. It can be seen that, with the density of the connection plates increasing from 800 to $11600 \mathrm{~kg} / \mathrm{m}^{3}$, the widths of the first and the second band gap vary slightly, while the width of the third band gap rises rapidly. For the first and second band gap, with the increase of the density of the connection plate, the stiffness of the connection plates in the 

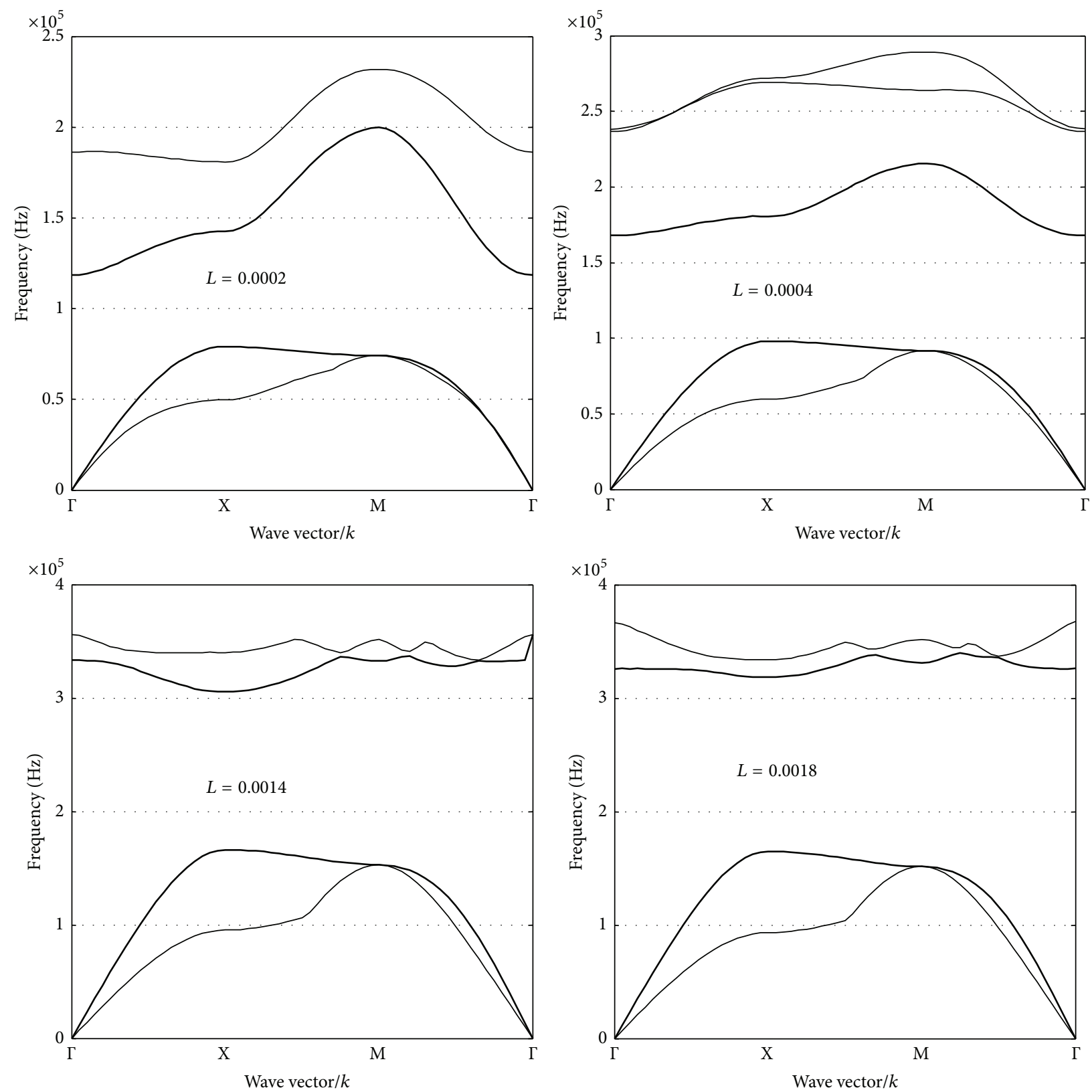

FIgURE 6: The dispersion curves of the phononic crystals with different length of connection plates structures.

mass-spring system increases rapidly, while the mass of the resonator has no change, resulting in the resonance frequency minor declining accordingly.

\section{Conclusions}

In this paper, the band structures of two-dimensional PCs with the microcavity structure are investigated using FEM. From these PC structures, a complete band gap can be obtained. The band gap is notably lowered, as expected. The microcavity structure formed with four connection plates bridge to the resonator possess many locally resonators lead to a low-frequency band gap. The displacement fields of eigenmodes are studied to reveal the physical mechanism for the existence of material's periodic arrangement on the lowfrequency band gaps. It was shown that the influence of the geometric dimension and connection plate adjacent shape is much higher than the influence of the materials properties. This provides a more important design factor in engineering application.

\section{Conflict of Interests}

The authors declare that there is no conflict of interests regarding the publication of this paper. 


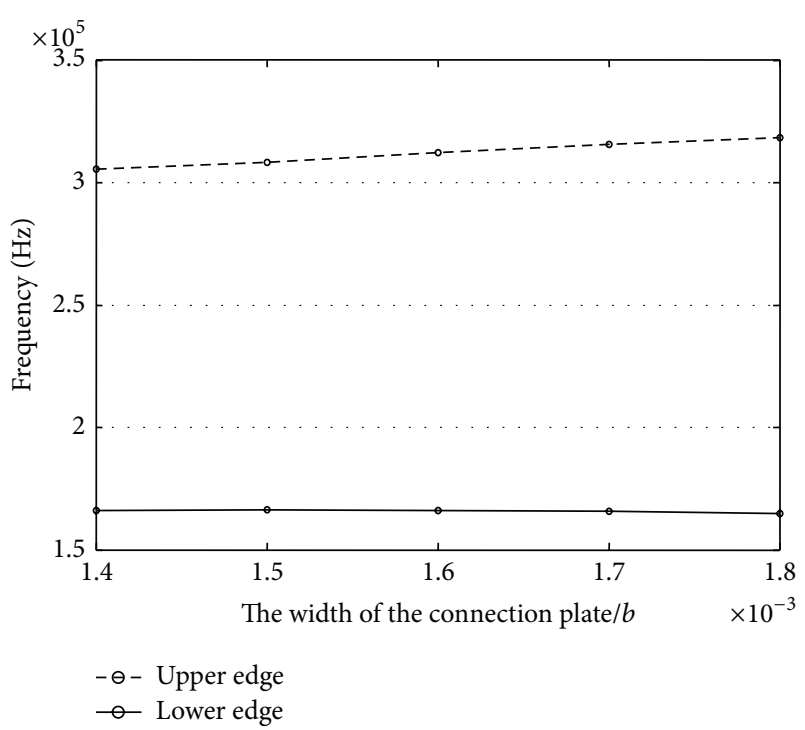

FIgURE 7: The evolution of the band gap as a function of the width of the connection plate $b$.

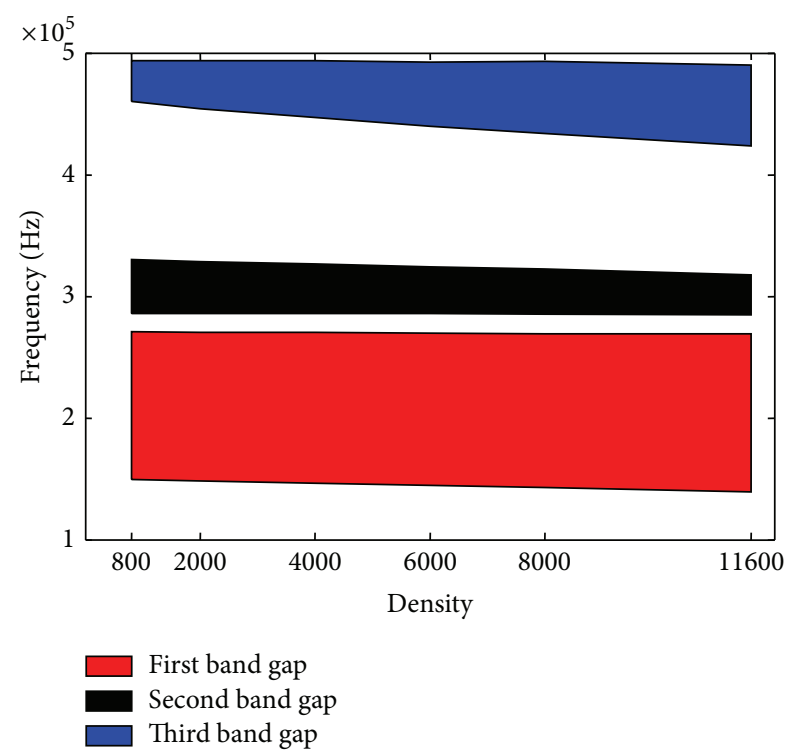

FIGURE 8: The evolution of the band gap as a function of the density of the connection plate.

\section{Acknowledgments}

The authors are grateful to the support from the National Science Foundation of China (no. 51175097) and the Zhejiang Provincial Natural Science Foundation for Excellent Young Scientists (no. LR13E050002). This work is also supported in part by the Innovation Project of GUET Graduate Education (no. XJYC2012003), which is very much appreciated.

\section{References}

[1] M. S. Kushwaha, P. Halevi, G. Martínez, L. Dobrzynski, and B. Djafari-Rouhani, "Theory of acoustic band structure of periodic elastic composites," Physical Review B, vol. 49, no. 4, pp. 23132322, 1994.
[2] Y. Huang, S. Liu, and J. Zhao, "Optimal design of twodimensional band-gap materials for uni-directional wave propagation," Structural and Multidisciplinary Optimization, vol. 48, no. 3, pp. 487-499, 2013.

[3] M. S. Kushwaha, P. Halevi, L. Dobrzynski, and B. DjafariRouhani, "Acoustic band structure of periodic elastic composites," Physical Review Letters, vol. 71, no. 13, pp. 2022-2025, 1993.

[4] M. Liu, J. Xiang, H. Gao, Y. Jiang, Y. Zhou, and F. Li, "Research on band structure of one-dimensional phononic crystals based on wavelet finite element method," CMES-Computer Modeling in Engineering \& Sciences, vol. 97, no. 5, pp. 425-436, 2014.

[5] Y. Chen, J. Li, J. Zhou, H. Tao, M. Zhou, and D. Y. Yu, "Phononic first band gap of quaternary layered periodic structure with the lumped-mass method," Shock and Vibration, vol. 2014, Article ID 189539, 9 pages, 2014.

[6] H. Policarpo, M. M. Neves, and A. M. R. Ribeiro, "Dynamical response of a multi-laminated periodic bar: analytical, numerical and experimental study," Shock and Vibration, vol. 17, no. 4-5, pp. 521-535, 2010.

[7] Y. Lai, X. Zhang, and Z.-Q. Zhang, "Engineering acoustic band gaps," Applied Physics Letters, vol. 79, no. 20, pp. 3224-3226, 2001.

[8] Y.-F. Wang, Y.-S. Wang, and L. Wang, "Two-dimensional ternary locally resonant phononic crystals with a comblike coating," Journal of Physics D: Applied Physics, vol. 47, no. 1, Article ID 015502, 2014.

[9] Z. Liu, X. Zhang, Y. Mao et al., "Locally resonant sonic materials," Science, vol. 289, no. 5485, pp. 1734-1736, 2000.

[10] W. Trabelsi, H. Franklin, A. Tinel, and S. Derible, "Band gap structures in underwater screens of periodically spaced porous plates," Ultrasonics, vol. 54, no. 4, pp. 1097-1103, 2014.

[11] K. L. Manktelow, M. J. Leamy, and M. Ruzzene, "Topology design and optimization of nonlinear periodic materials," Journal of the Mechanics and Physics of Solids, vol. 61, no. 12, pp. 2433-2453, 2013.

[12] H. Salehi, M. Aryadoust, and M. Z. Shoushtari, "Investigation the effect of lattice angle on the band gap width in 3D phononic crystals with rhombohedral(I) lattice," Applied Physics A: Materials Science and Processing, vol. 116, no. 1, pp. 69-77, 2014.

[13] R. Sainidou and N. Stefanou, "Guided and quasiguided elastic waves in phononic crystal slabs," Physical Review B: Condensed Matter and Materials Physics, vol. 73, no. 18, Article ID 184301, 9 pages, 2006.

[14] M. Oudich, Y. Li, B. M. Assouar, and Z. Hou, "A sonic band gap based on the locally resonant phononic plates with stubs," New Journal of Physics, vol. 12, Article ID 083049, 2010.

[15] K. Yu, T. Chen, and X. Wang, "Large band gaps in twodimensional phononic crystals with neck structures," Journal of Applied Physics, vol. 113, no. 13, Article ID 134901, 2013.

[16] J.-C. Hsu, "Local resonances-induced low-frequency band gaps in two-dimensional phononic crystal slabs with periodic stepped resonators," Journal of Physics D: Applied Physics, vol. 44, no. 5, Article ID 055401, 2011.

[17] Y. Cheng, X. J. Liu, and D. J. Wu, "Band structure of a phononic crystal plate in the form of a staggered-layer structure," Journal of Applied Physics, vol. 109, no. 6, Article ID 064904, 2011. 

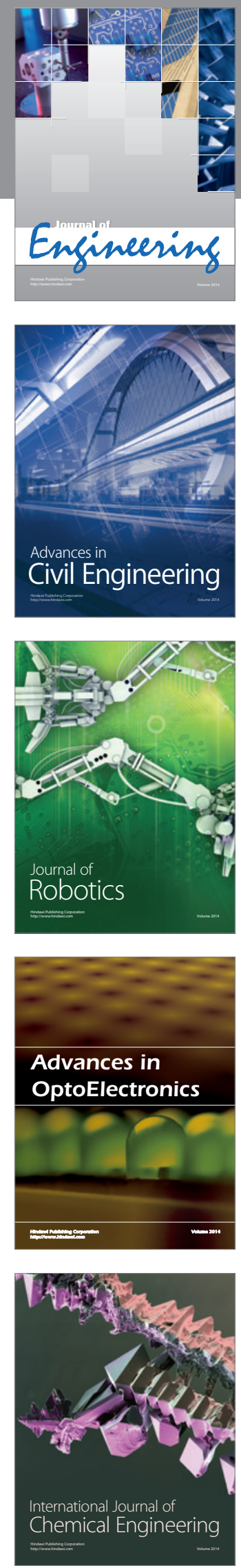

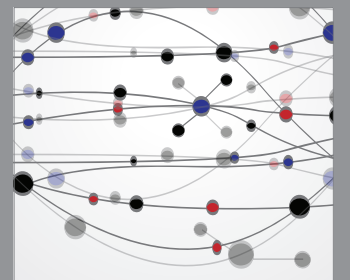

The Scientific World Journal
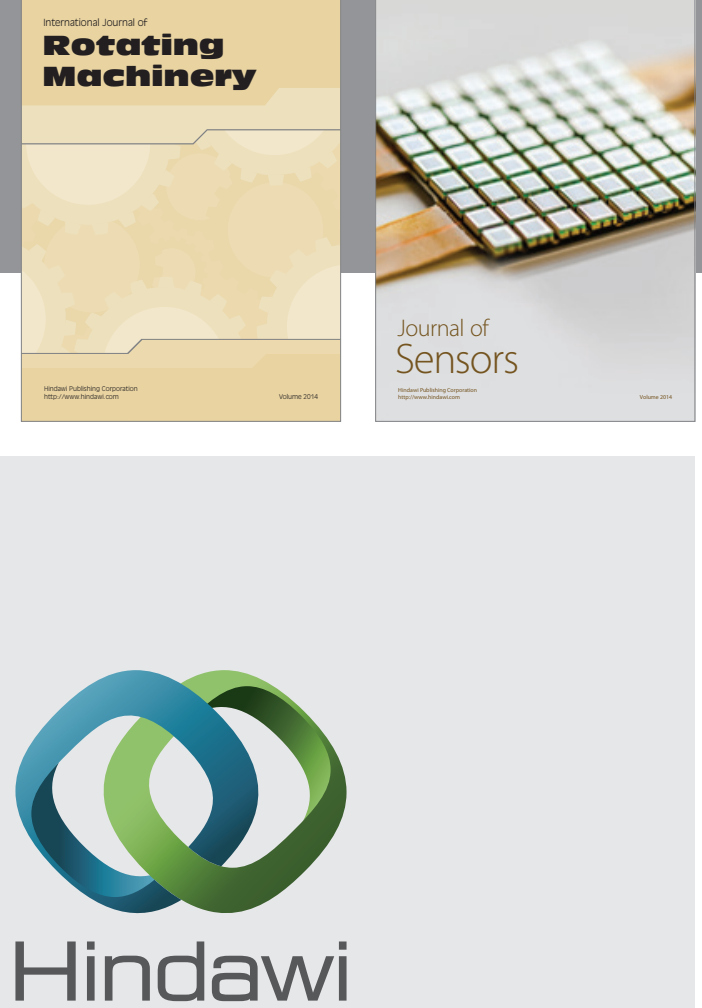

Submit your manuscripts at http://www.hindawi.com
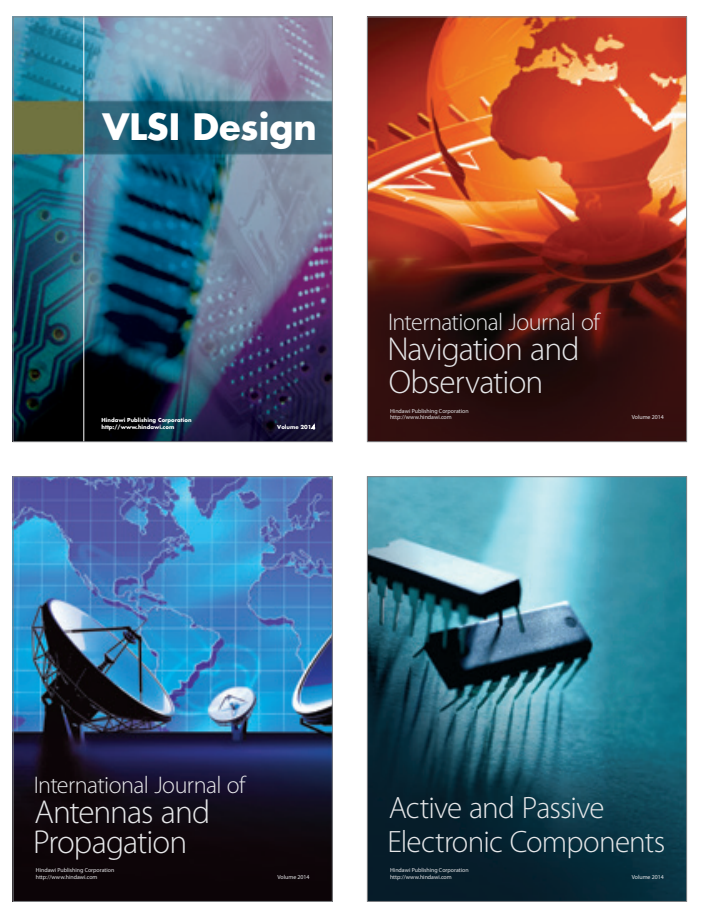
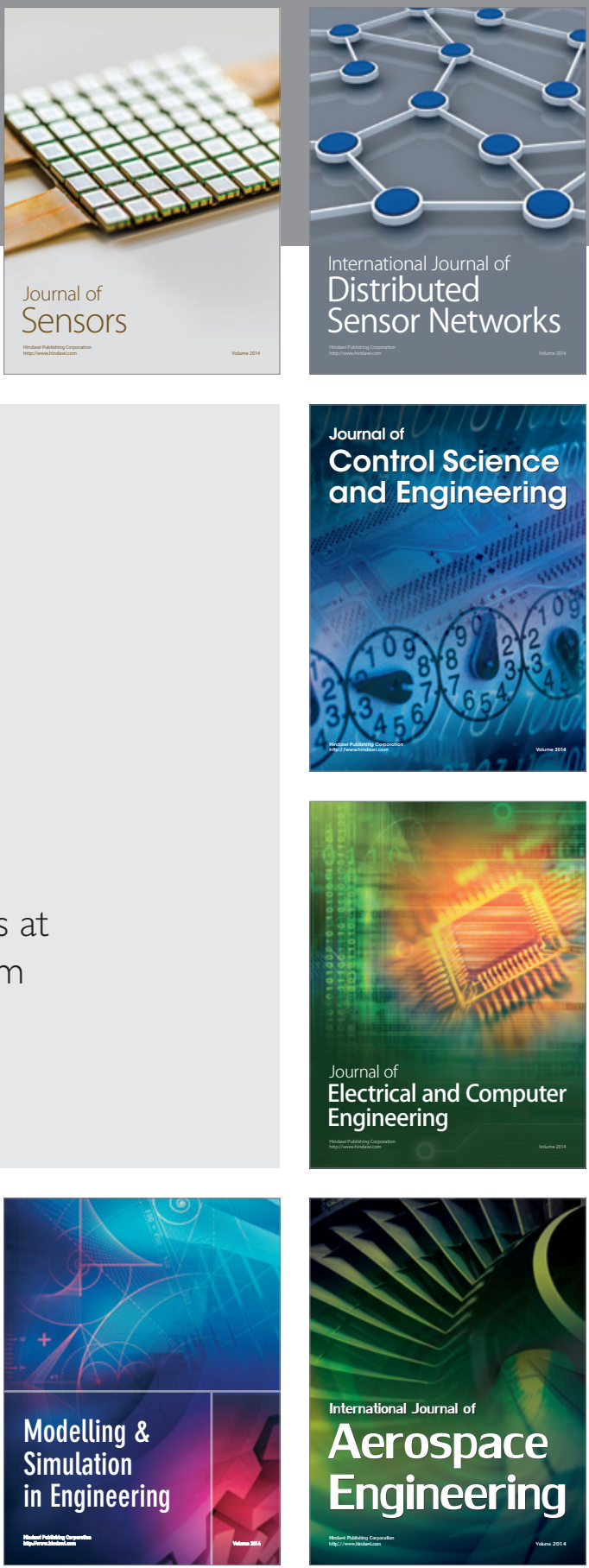

Journal of

Control Science

and Engineering
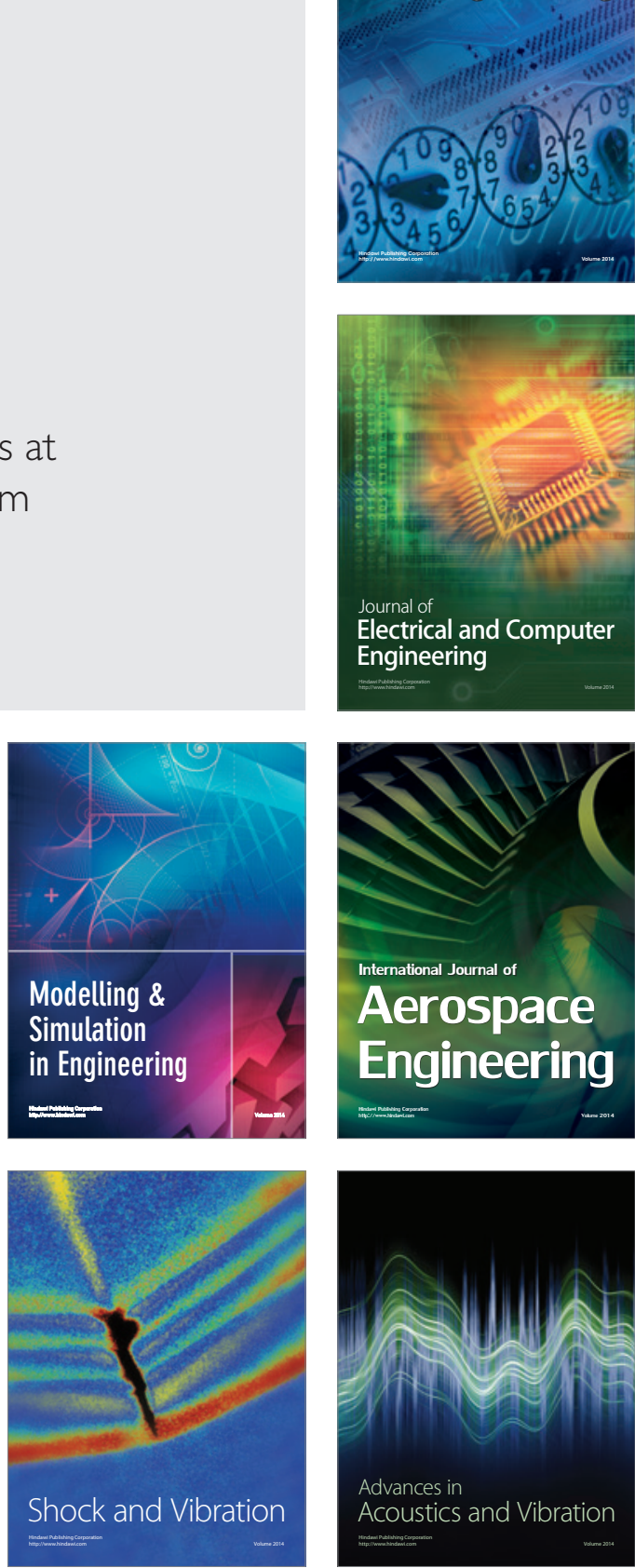